\title{
Adding Blood to St Thomas Solution Does Not Improve Mortality in Pediatric Cardiac Surgery: A Meta-analysis of a Homogenous Population
}

\author{
Mark Awori, Nikita Mehta, Naomi Kebba, Enoch Makori \\ University of Nairobi
}

Correspondence to: Dr. Mark Awori; Email: mnawori@yahoo.com

\begin{abstract}
Background: Cardioplegia is the gold standard for providing ideal operating conditions while effecting myocardial protection. Some studies suggest that adding blood to St Thomas cardioplegia solution improves efficacy; this is generally accepted as true. However, the few meta-analyses conducted on children have pooled heterogeneous populations; this raises concern about the validity of their conclusions. Methods: PUBMED, the Cochrane Library and Google Scholar were searched systematically until March 2019 using the search terms "cardioplegia"; "myocardial protection"; "pediatric" "pediatric"; "children"; "infants"; "neonates". Full text articles were examined if abstracts revealed that the studies possibly contained a blood cardioplegia arm and a crystalloid cardioplegia arm. Studies were included in the meta-analysis if they had a 4:1 blood cardioplegia arm and a St Thomas solution arm. Meta-analysis was performed using Meta-Mar software.
\end{abstract}

Results: The search retrieved 423 articles; 5 were included in the meta-analysis, representing 324 patients. The risk ratio for operative mortality was $0.77(95 \%$ CI $0.24-2.5 ; \quad p=0.66$ ). Little evidence was seen of heterogeneity of the pooled patients. Conclusion: Adding blood to St Thomas cardioplegia solution did not improve in-hospital operative mortality; this may have implications for use of blood cardioplegia.

Keywords: Cardioplegia, Pediatric, Congenital heart surgery, Mortality, Meta-analysis

Ann Afr Surg. 2020; 17(2):60-64

DOI: http://dx.doi.org/10.4314/aas.v17i2.4

Conflicts of Interest: None

Funding: None

(C) 2020 Author. This work is licensed under the Creative Commons Attribution 4.0 International License.

\section{Introduction}

The attainment of optimal cardiac surgical results requires the heart to be still, flaccid, lying in a bloodless field and protected from irreversible ischemic damage. The gold standard for achieving this is the use of cardioplegia, which employs three principle concepts for myocardial protection: chemical arrest, hypothermia and additional protection such as manipulating ionic imbalance (1). The merits and demerits of the use of various types of cardioplegia have been examined; however, ambiguity still remains regarding which is best $(2,3)$. Although the exact mechanism of action remains unknown, the addition of blood to a crystalloid cardioplegia base is thought to improve myocardial protection by prolonging aerobic metabolism (4). In comparing clinical outcomes between blood and nonblood cardioplegia, most randomized clinical trials

have been on adult patients; pediatric trials are few (2). Some systematic reviews with meta-analyses have been conducted; however, the populations included have been significantly heterogeneous (5). Meta-analysis is a powerful and useful tool when correctly applied; elemental to proper application is the analysis of populations that are as homogenous as possible (6). Available studies examining surgical outcomes related to cardioplegia type are too heterogeneous for conducting meta-analyses (3). To optimize homogeneity, answers to the following questions may guide decisions on which studies to include in a meta-analysis:

1. Is the study on pediatric or adult patients?

2. Did cardioplegia achieve depolarized or hyperpolarized arrest?

3. Was blood added to the cardioplegia solution? 
4. Was warm or cold cardioplegia used?

5. What cardioplegia dosing regimen was used?

6. Were other modifications to or variants of cardioplegia used?

Studies for inclusion should differ in as few parameters as possible, preferably in only one (6). The current study aimed to determine whether blood-based cardioplegia was associated with a lower in-hospital operative mortality than non-blood-based cardioplegia in pediatric cardiac surgery. The results of this study may have implications for the use of blood cardioplegia in general.

\section{Methods}

\section{Search strategy and selection criteria}

Using the PRISMA-P checklist (7), PUBMED, the Cochrane Library and Google Scholar were searched systematically until March 2019 using the search terms "cardioplegia"; "myocardial protection"; "pediatric" "pediatric"; "children"; "infants"; "neonates". Table 1 shows the search strategies.

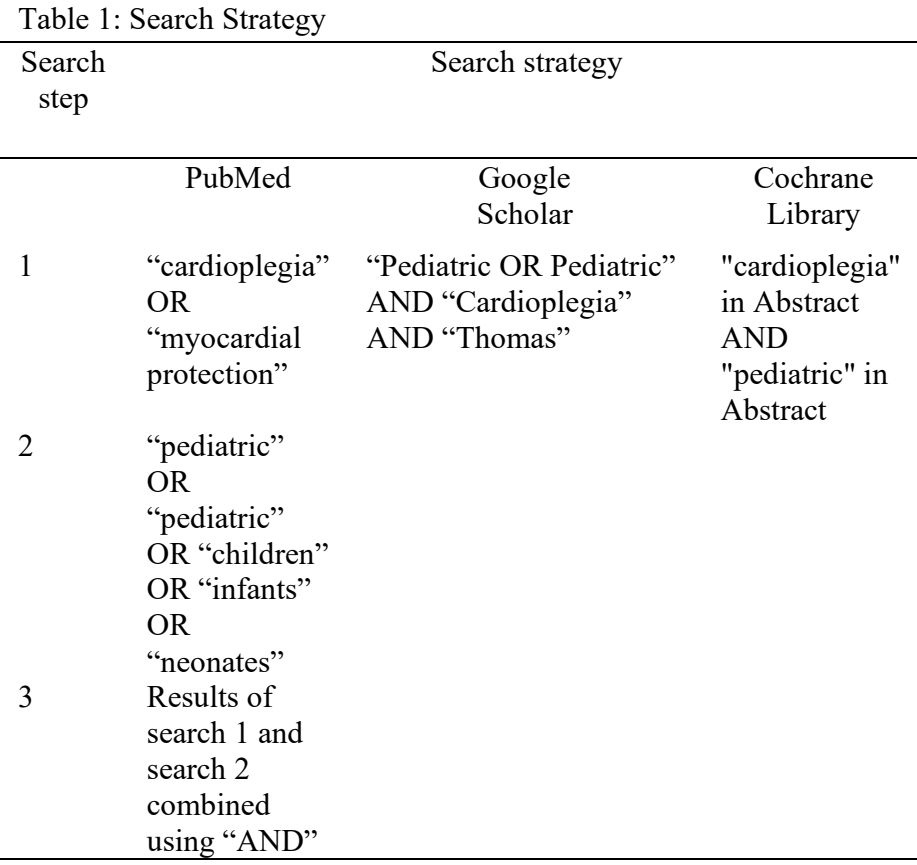

Titles and abstracts were interrogated and full-text articles were obtained when the abstract showed a possibility that the study included a group of pediatric patients exposed to blood cardioplegia and a group of pediatric patients exposed to non-blood cardioplegia ( $\mathrm{St}$ Thomas solution). The outcome of interest was inhospital operative mortality. Non-English language studies and non-human studies were excluded. Only randomized clinical trials were included. The six questions mentioned above were used to refine the choice of studies for inclusion: only studies that used intermittent cold cardioplegia (blood or non-blood) were included and only after all authors were in agreement about their inclusion. Two reviewers performed the search and data extraction; when necessary, discrepancies were resolved by discussion and consensus with a third reviewer. Figure 1 shows the search flow.

423 articles retrieved

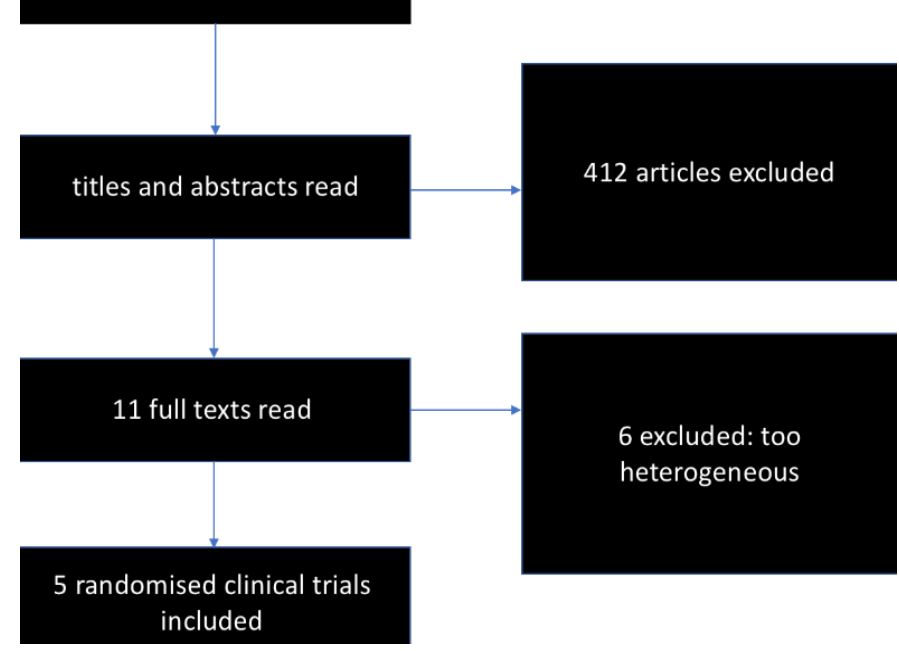

Figure 1: Search flow diagram

\section{Statistical analysis}

The meta-analysis used Meta-Mar free online metaanalysis service (V1.8.0) (8); risk ratio (RR) was used for analysis. Fixed and random effects moDels were employed; I2-statistic was used to assess for heterogeneity (6).

\section{Results}

The search yielded 423 articles; 11 relevant full-texts were obtained after examining titles and abstracts. Six full-text articles were rejected on grounds of unacceptable clinical heterogeneity (according to the six questions). Table 2 shows the important details of the 5 studies included in the meta-analysis and Table 3 lists the full text articles rejected.

Table 2: Studies included on meta-analysis

\begin{tabular}{llll}
\hline Study & $n(324)$ & Cardioplegia type & $\begin{array}{l}\text { Dose } \\
\text { timing } \\
(\mathrm{min})\end{array}$ \\
\hline Young 1997 (9) & 138 & B: St-Th-1(4:1) & $20-30$ \\
Caputo 2002 (10) & 40 & B: St-Th-1(4:1) & $20-30$ \\
Modi 2004 (11) & 46 & B: St-Th-1(4:1) & $20-30$ \\
Amark 2005 (12) & 30 & B: St-Th-1 OR & $20-30$ \\
& & 2(4:1) & \\
Romolo 2019 (13) & 70 & B: St-Th-1(4:1) & 20 \\
\hline B=blood; St-Th=St Thomas &
\end{tabular}


Table 3: List of excluded full text papers

\begin{tabular}{lll}
\hline & Authors & Year \\
\hline 1. & Sobieraj et al. (14) & 2018 \\
2. & O'Brien et al. (15) & 2009 \\
3. & Sinha et al. (16) & 2008 \\
4. & Zhu et al. (17) & 2006 \\
5. & Modi et al. (18) & 2006 \\
6. & Toyoda et al. (19) & 2003 \\
\hline
\end{tabular}

Table 4 shows the key findings of the meta-analysis and Figure 2 the forest plot.

\begin{tabular}{llllll}
\multicolumn{6}{l}{ Table 4: Meta-analysis results } \\
\hline $\begin{array}{l}\text { Risk } \\
\text { ratio }\end{array}$ & SE & $95 \%$ CI & p-value & $\mathrm{I}^{2}$ \\
\hline Fixed effect & 0.77 & 0.6 & $0.24-2.5$ & 0.66 & $0 \%$ \\
Random effect & 0.51 & 0.59 & $0.16-1.63$ & 0.25 & $0 \%$ \\
\hline
\end{tabular}

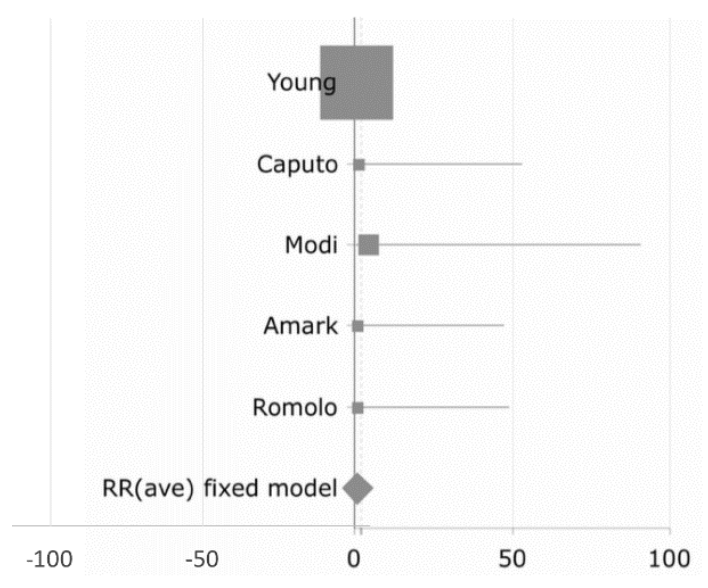

Figure 2: Forest plot

\section{Discussion}

Del Nido solution is composed of 1-part blood and 4 parts base solution (i.e., it is $20 \%$ blood). It is generally administered as single dose and Delivers arrest for up to 90 minutes. Del Nido solution, and blood cardioplegia in general, is the preferred option for some practitioners in pediatric cardiac surgery based on evidence such as that provided by a relatively recent randomized clinical trial by Talwar et al. (20). This study found that the Del Nido solution improved postoperative outcomes in cardiac output, duration of mechanical ventilation, troponin-1 release and electron microscopic evidence of myocardial damage (20). Although these are probably more sensitive outcome measures, we choose not to examine metabolic markers of myocardial protection as they were not measured in a homogenous fashion. For similar reasons we did not examine postoperative inotrope use. Simplicity improves efficiency and reduces the chance of error. In this regard, minimizing the steps required to execute any stage of cardiac surgery has a cumulative effect, and marginal gains such as these, have been shown to result in significant improvements in outcomes in other disciplines (21). If adding blood to cardioplegia does not improve outcomes, it should not be added. The ultimate measure of the success of cardiac surgery is long-term mortality. Before long-term mortality can be considered, operative mortality must be examined. Although outcomes such as postoperative cardiac index and release of metabolic markers are considered to be more sensitive markers of the degree of myocardial preservation, they cannot be assumed to translate into improvements in the ultimate outcome. Furthermore, the best quality evidence on the effect of adding blood to cardioplegia, on operative outcome, must be documented. Our work attempts to document the available evidence of the highest quality in pediatric cardiac surgery. As we worked to achieve this, we remained aware that the immature heart responds differently to ischemia and that the transition phase is around 3 months of age (22).

Results of clinical studies should be easily applied to patient care; we chose to examine risk ratio (RR) instead of odds ratio (OR) as it is a more intuitive parameter for clinicians (23). We found that there was no significant difference, in in-hospital operative mortality, between blood and non-blood (St Thomas crystalloid solution) cardioplegia in pediatric cardiac surgery. A relatively large meta-analysis examining 5576 patients from 36 RCTs found that blood cardioplegia was not superior to crystalloid (non-blood) cardioplegia for myocardial protection with respect to in-hospital operative mortality (2). However, the meta-analysis included a highly heterogenous group of patients; no distinction was made between children and adults; use of warm cardioplegia and cold cardioplegia; and use of continuous cardioplegia Delivery, intermittent Delivery and single dose Delivery. Two relatively recent meta-analyses $(5,24)$ comparing blood and non-blood cardioplegia in pediatric cardiac surgery found no difference in clinical outcomes following the use of either strategy. Of particular interest to us was that Mylonas et al. found no difference in 30day mortality (5). Fang et al. examined metabolic markers of myocardial protection, length of postoperative ventilation and length of intensive care unit (ICU) stay; they found that these outcomes were essentially the same irrespective of strategy used (18). The study by Mylonas was solely on pediatric patients; however, it included studies using warm cardioplegia, cold cardioplegia, intermittent dosing and single dosing, and it also included studies with other significant potential confounders. For 
example, it included a study by Mimic et al. (25) which had a high concentration of glucose in one arm (25). The study by Fang et al. was more clinically homogenous, but it included a study that compared warm blood cardioplegia with cold non-blood cardioplegia (24). The heterogeneity in these two meta-analyses could have affected the pooled outcome. When the six questions are used to assess study inclusion in these two meta-analyses, it can be argued that the studies included were too heterogeneous for meta-analysis. This sentiment was echoed recently by Drury et al. who concluded that cardioplegia studies in pediatric patients were too heterogeneous for meaningful meta-analyses (3).

Study heterogeneity may be fundamentally classified as heterogeneity caused by chance and heterogeneity caused by systematic factors; the degree of heterogeneity may simply be assessed clinically or statistically (6). Ensuring clinical homogeneity is a pragmatic method for appropriately selecting studies for inclusion in metaanalyses; the six questions mentioned above facilitate this for cardioplegia strategy comparison (26). It is standard practice to assess statistical heterogeneity when conducting meta-analyses. In the current study, we sought to minimize the clinical heterogeneity as much as possible by only including studies using intermittent cold cardioplegia. For the same reason, we excluded studies that looked at endpoints other than mortality. By doing so, we tried to reduce any confounding resulting from differences in cardioplegia dosing, cardioplegia temperature, and cardioplegia additives other than blood. In particular, we included only studies that had a blood cardioplegia ratio of $4: 1$; blood to crystalloid. Statistically, our studies heterogeneity was low $\left(\mathrm{I}^{2}=0 \%\right)$. The validity of this finding is strengthened by the similar RR results obtained by the fixed and random effect moDels in our study (27).

Meta-analyses are susceptible to bias; of particular concern are selection bias and small study bias (28). Although it is impossible to determine with certainty the extent to which bias has affected pooled results, a sensible attempt to quantify bias using a funnel plot is performed when appropriate (29). We did not construct a funnel plot as its benefit is questionable when less than 10 studies are included in the analysis (29). We used the meta-mar online calculator which has been validated against RevMan and is significantly easier to use (8). Four out of five of the studies that we included had no mortality (zero-event) in at least one of their arms; three studies had no mortality in both arms. A continuity correction is usually used for analysis when there is no event (e.g. mortality) in an arm. It has been shown that a continuity correction of 0.5 reduces bias when there is no event (e.g. mortality) in both arms, particularly if there is a greater likelihood of there being no significant difference in outcomes between arms (27). A large observational study of over 7000 pediatric patients (greater than 3000 patients in each arm) found no difference in in-hospital operative mortality between Del Nido (single-dose blood-based cardioplegia) and St Thomas II solution (crystalloid cardioplegia) (30); in view of this we opted to use a continuity correction of 0.5 . It has been recommended that zero-event studies be included in meta-analyses when it is likely that there is no difference between interventions. We think that the findings of this large observational study provide further justification for including zero-event (i.e. no mortality) studies in our meta-analysis (27). Modi et al. suggested that blood cardioplegia offers better results in cyanotic patients (11). There was no significant difference in the proportion of cyanotic patient, in our study, when all cases, except those in the study by Romolo et al. (13), were pooled together. Although Romolo et al. did not indicate the proportion of cyanotic patients in each arm, their study was on patients with tetralogy of Fallot. The mean arterial oxygen saturation was less than $90 \%$ in both arms and was not significantly different; the operative mortality was the same in both arms. When this fact, and the fact that the combined proportions of cyanotic patients in the other four studies were not significantly different in the two arms, it would seem that there is no advantage of using blood cardioplegia in cyanotic patients.

\section{Conclusion}

Our results suggest that the in-hospital operative mortality associated with the addition of blood to St Thomas I or II solutions in pediatric cardiac surgery is not significantly different from that associated with the use of non-blood (St Thomas solution I or II) cardioplegia. Appropriately conducted meta-analyses on well-designed RCTs remain the highest form of evidence with respect to clinical practice. We conducted a metaanalysis, in accordance with best practice guiDelines, of available RCTs that were pragmatically clinically homogenous.

We ensured that both cardioplegia arms used cardioplegia that caused depolarized arrest and that the only difference between the two arms was the addition of blood in equal 
concentrations to one arm. Our results may have implications for the use of blood cardioplegia. RCTs with larger populations are required to confirm our finding. We recommend that additional well-designed RCTs be conducted to systematically and robustly examine the role of adding blood to cardioplegia.

\section{References}

1. Chambers DJ, Hearse DJ. Developments in cardio-protection: "Polarized" arrest as an alternative to "depolarized" arrest. Ann Thorac Surg 1999; 68:1960-6.

2. Sá MPBO, Rueda FG, Ferris PE, et al. Is there any difference between blood and crystalloid cardioplegia for myocardial protection during cardiac surgery? A meta-analysis of 5576 patients from 36 randomized trials. Perfusion. 2012; 27:535-46.

3. Drury NE, Yam I, Patel AJ, et al. Cardioplegia in paediatric cardiac surgery: A systematic review of randomized controlled trials. Interact CardioVasc Thorac Surg. 2019; 28:144-50.

4. Buel ST, Striker CW, O'Brien JE. Del Nido versus St. Thomas cardioplegia solutions: A single-center retrospective analysis of post cross-clamp defibrillation rates. JECT. 2016; 48:67-70.

5. Mylonas KS, Tzani A, Metaxas P, et al. Blood versus crystalloid cardioplegia in pediatric cardiac surgery: A systematic review and meta-analysis. Pediatr Cardiol. 2017; 38:1527-39.

6. Melsen WG, Bootsma MC, Rovers MM et al. The effects of clinical and statistical heterogeneity on the predictive values of results from meta-analyses. Clin Microbiol Infect. 2014; 20:123-9.

7. Moher D, Shamseer L, Clarke M, et al. Preferred reporting items for systematic review and meta-analysis protocols (PRISMA-P) 2015 statement. Systematic Reviews. 2015; 4:1.

8. Meta-Mar free online meta-analysis service. Available at http://www.meta-mar.com . Accessed April 11, 2019.

9. Young JN, Choy IO, Silva NK, et al. Antegrade cold blood cardioplegia is not demonstrably advantageous over cold crystalloid cardioplegia in surgery for congenital heart disease. J Thorac Cardiovasc Surg. 1997; 114:1002-9.

10. Caputo M, Modi P, Imura H, et al. Cold blood versus cold crystalloid cardioplegia for repair of ventricular septal defects in pediatric heart surgery: A randomized controlled trial. Ann Thorac Surg. 2002; 74:530-5.

11. Modi P, Suleiman MS, Reeves B, et al. Myocardial metabolic changes during pediatric cardiac surgery: A randomized study of 3 cardioplegic techniques. J Thorac Cardiovasc Surg. 2004; 128: $67-75$.

12. Amark K, Berggren H, Bjork K, et al. Blood cardioplegia provides superior protection in infant cardiac surgery. Ann Thorac Surg. 2005; 80:989-94.

13. Romolo H, Hernisa L, Fakhri D, et al. Comparison between blood and non-blood cardioplegia in tetralogy of Fallot. Asian Cardiovasc Thor Annals. 2019; 27(2):75-9.

14. Sobieraj M, Kilanowska M, Ładziński P, etal. Type of cardioplegic solution as a factor influencing the clinical outcome of open-heart congenital procedures. Kardiochir Torakochirurgia Pol. 2018; 15(2):86-94.

15. O'Brien JD, Howlett SE, Burton HJ, et al. Pediatric cardioplegia strategy results in enhanced calcium metabolism and lower serum troponin T. Ann Thorac Surg. 2009; 87(5):1517-23.

16. Sinha P, Zurakowski D, Jonas RA. Comparison of two cardioplegia solutions using thermodilution cardiac output in neonates and infants. Ann Thorac Surg. 2008; 86(5):1613-9.

17. Zhu DM, Wang W, Xu ZW et al. Seven years' experience of pediatric cardiopulmonary bypass: 8685 cases in Shanghai Children's Medical Center. ASAIO J. 2006; 52(5):556-8.

18. Modi P, Suleiman SM, Reeves BC, et al. Changes in myocardial free amino acids during pediatric cardiac surgery: A randomized controlled trial of three cardioplegic techniques. Eur J Cardiothorac Surg. 2006; 30(1):41-8.

19. Toyoda Y, Yamaguchi M, Yoshimura N, et al. Cardioprotective effects and the mechanisms of terminal warm blood cardioplegia in pediatric cardiac surgery. J Thorac Cardiovasc Surg. 2003; 125(6):1242-51.

20. Talwar S, Bhoje A, Sreenivas V, et al. Comparison of Del Nido and St Thomas cardioplegia solutions in pediatric patients: A prospective randomized clinical trial. Semin Thorac Cardiovasc Surg. 2017; 29(3):366-74.

21. Clear J. This coach improved every tiny thing by 1 percent and here's what happened. https://jamesclear.com/marginal-gains.

22. Onwugbufor M, Levy RJ, Zurakowski D, et al. Myocardial cytochrome oxidase activity increases with age and hypoxemia in patients with congenital heart disease. Perfusion. 2017; 32(4):306-12.

23. O'Connor AM. Interpretation of odds and risk ratios. J Vet Intern Med. 2013; 27:600-3.

24. Fang Y, Long C, Lou S, et al. Blood versus crystalloid cardioplegia for pediatric cardiac surgery: A meta-analysis. Perfusion. 2015; 30:529-36.

25. Mimic B, Ilic S, Vulicevic I, et al. Comparison of high glucose concentration blood and crystalloid cardioplegia in paediatric cardiac surgery: A randomized clinical trial. Interact Cardiovasc Thorac Surg. 2016; 22(5):553-60.

26. Israel H, Richter RR. A guide to understanding meta-analysis. J Orthop Sports Phys Ther. 2011; 41:496-504.

27. Cheng J, Pullenayegum E, Marshall JK, et al. Impact of including or excluding both-armed zero-event studies on using standard meta-analysis methods for rare event outcome: A simulation study. BMJ Open. 2016; 6:e010983.

28. Greco T, Zangrillo A, Biondi-Zoccai G, et al. Meta-analysis: Pitfalls and hints. Heart, Lung and Vessels. 2013; 5(4):219-25

29. Sterne JA, Sutton AJ, Ioannidis JP. Recommendations for examining and interpreting funnel plot asymmetry in metaanalyses of randomized controlled trials. BMJ. 2011;343: d4002.

30. Zhu D, Wang W, Xu Z, et al. Seven years' experience of pediatric cardiopulmonary bypass: 8685 cases in Shanghai Children's Medical Center. ASAIO Journal. 2006; 52:556-8. 